\title{
New pathways for pressure and force sensor systems - Strain sensing with nanogranular metals
}

\author{
Dr. Christian H. Schwalb ${ }^{1,3}$, Prof. Dr. Michael Huth ${ }^{1}$, Prof. Dr. Friedemann Völklein ${ }^{2}$, Dr. \\ Alexander Kaya ${ }^{3}$ \\ ${ }^{1}$ Physikalisches Institut, Goethe Universität, Max-von-Laue-Str. 1, 60438 Frankfurt, Tel: 069-79847222 \\ ${ }^{2}$ RheinMain University of Applied Sciences, Am Brückweg 26, 65428 Rüsselsheim, Tel: 06142-898-4531 \\ ${ }^{2}$ NanoScale Systems GmbH, Robert-Bosch-Str. 7, 64293 Darmstadt, Tel: 06151-6674037 \\ E-Mails: schwalb@nanoss.de, michael.huth@physik.uni-frankfurt.de, friedemann.voelklein@hs-rm.de, \\ kaya@nanoss.de
}

\begin{abstract}
This paper introduces a novel methodology for the fabrication of threedimensional (3D) strain sensing elements for micro- and nanoelectromechanical applications based on the tunneling effect in nanogranular metals. The nanogranular tunneling resistors (NTR) are prepared by the direct writing technique of focused electron-beam-induced deposition, a process which enables the preparation of sensor structures well below $20 \mathrm{~nm}$ on a great variety of substrates and different materials. We use a cantilever-based deflection technique to determine the sensitivity (gauge factor) of the sensor element. The sensitivity and the resistance can be altered either by the thickness of the deposits, by post electron-beam irradiation, or by passivation with an amorphous silicon layer. We demonstrate applications for the NTR sensor elements in the field of cantilever- and membrane-based sensor systems and discuss possible future applications in MEMS/NEMS-technology.
\end{abstract}

\section{Introduction}

In recent years, the field of micro- and nanoelectromechanical systems (MEMS and NEMS) has shown a rapid progress as enabling technology for sensor device development based on the increasing demand for a continuous down-scaling of sensor functions in a broad variety of different application fields. For the realization of nano- and microscale strain/stress measurements different approaches have been followed, ranging from well-established methods, e.g., optical and piezoresistive [1, 2], to methods still in their initiation, e.g., diamond like carbon films (DLC) [3, 4], carbon nanotubes (CNT) [5, 6], and nanowires [7, 8]. In this work, we present a new methodology for the fabrication of strain-sensing elements based upon nanogranular metals. A specific strength of this methodology is the easy, single step fabrication procedure that is readily applicable for various force based sensor applications. For the fabrication of the nanogranular tunneling resistors (NTR) the focused electron-beam-induced deposition (FEBID) technique is used. This process is a mask-less bottom up technique that can be used for the creation of micro- and nanostructures in one, two and three dimensions based on the electron-beam-induced dissociation of a (metal-) organic precursor gas that is introduced locally in close proximity to a substrate surface (Figure 1). It is applicable to nearly all surfaces leading to a wide area of new applications. In addition, the high lateral resolution of the FEBID process allows for easy down-scaling of sensor structures well below $20 \mathrm{~nm}$. Depending on the choice of precursor and depostion conditions, the deposits can be either amorphous, poly-crystalline or nanogranular.[9, 10] 
For strain-sensing applications the nanogranular metals are of special interest. They represent artificial materials consisting of metallic nanocrystallites randomly dispersed in an insulating carbonaceous matrix. Their microscopic charge transport properties are dominated by tunneling between neighbored metallic nanoparticles, a process that is strongly influenced by correlation effects. These fundamental processes can be used for strain-sensing applications, since the tunnel coupling has an intrinsically exponential dependence on the distances between the neighbored metallic grains that are altered under mechanical strain.[11, 12, 13] Therefore nanogranular metals can be used for the development of new pressure and force sensor systems.

In the present work we systemetically study the strain sensing abilities of nanogranular metals using Pt-C based deposits. The NTR sensors elements show a highly linear response in cantilever based deflection measurements with highly reproducible gauge factors up to 16 . The noise of the NTRs follows a 1/f-frequency dependence hitting the Johnson noise level at about 1 $\mathrm{kHz}$. We discuss the theoretical achievable resolution limit for our NTR sensor elements and show practical applications for ultra small cantilevers and pressure membranes.

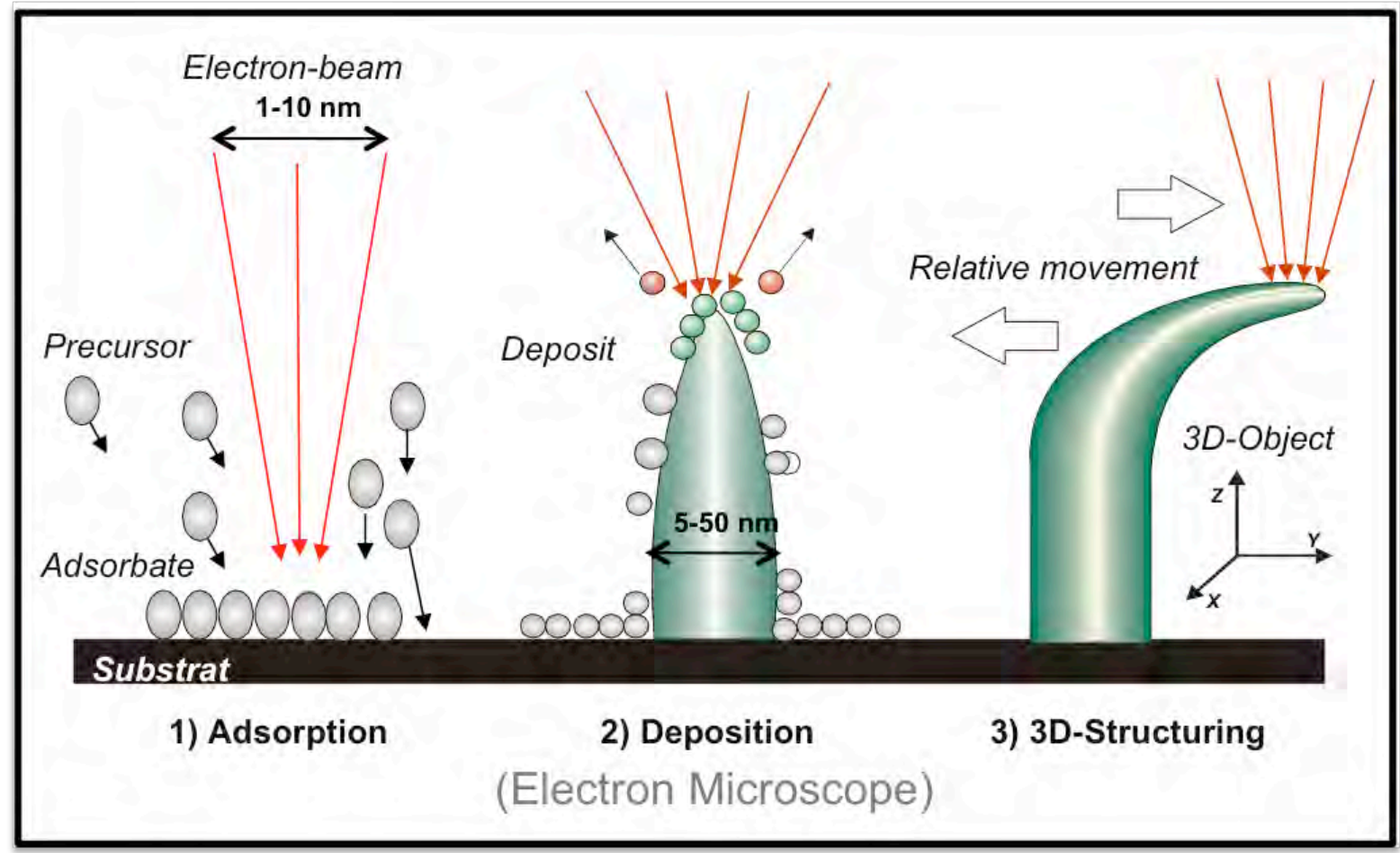

Figure 1: Schematic of the FEBID process inside a scanning electron microscope. The precursor gas is introduced via a gas injection system in close proximity to the focus of the electron beam and forms an adsorbate layer on the substrate (1). The electron beam dissociates the precursor molecules forming the nano-granular deposit with high resolution (2). A movement of the electron-beam or the substrate during the deposition process enables real 3D-structuring of the deposit (3).

\section{Experimental Section}

The NTR sensor elements were fabricated by FEBID inside a scanning electron microscope (SEM) using a dual-beam SEM/FIB microscope (FEI, Nova Nanolab 600) with a Schottky electron emitter and an ultimate resolution of $1 \mathrm{~nm}$. The precursor gases were introduced into the chamber via gas injection systems that are equipped with a capillary of $0.5 \mathrm{~mm}$ diameter which can be positioned in close proximity to the focus of the electron beam. The precursor gas forms an adsorbate layer on the substrate surface. The focused electron-beam then dissociates the precursor molecules forming volatile and non-volatile products. The non-volatile products form the nanogranular deposits. Due to the high resolution of the focused electron-beam 
structures well below $20 \mathrm{~nm}$ are feasible. In addition the FEBID technique is a mask-less single step process that can be applied to a great variety of substrates (such as glasses, oxydes, semiconductors, metals, polymers and more) and is not solely limited to silicon, which is used in conventional lithography techniques. When the electron-beam, or the substrate respectively, is moved in a controlled manner during the FEBID-deposition process real 3D-structuring is possible, giving the user a wide degree of freedom for the realization of even unorthodox and complex three-dimensional geometrical sensor structures (see Figure 1 for details).

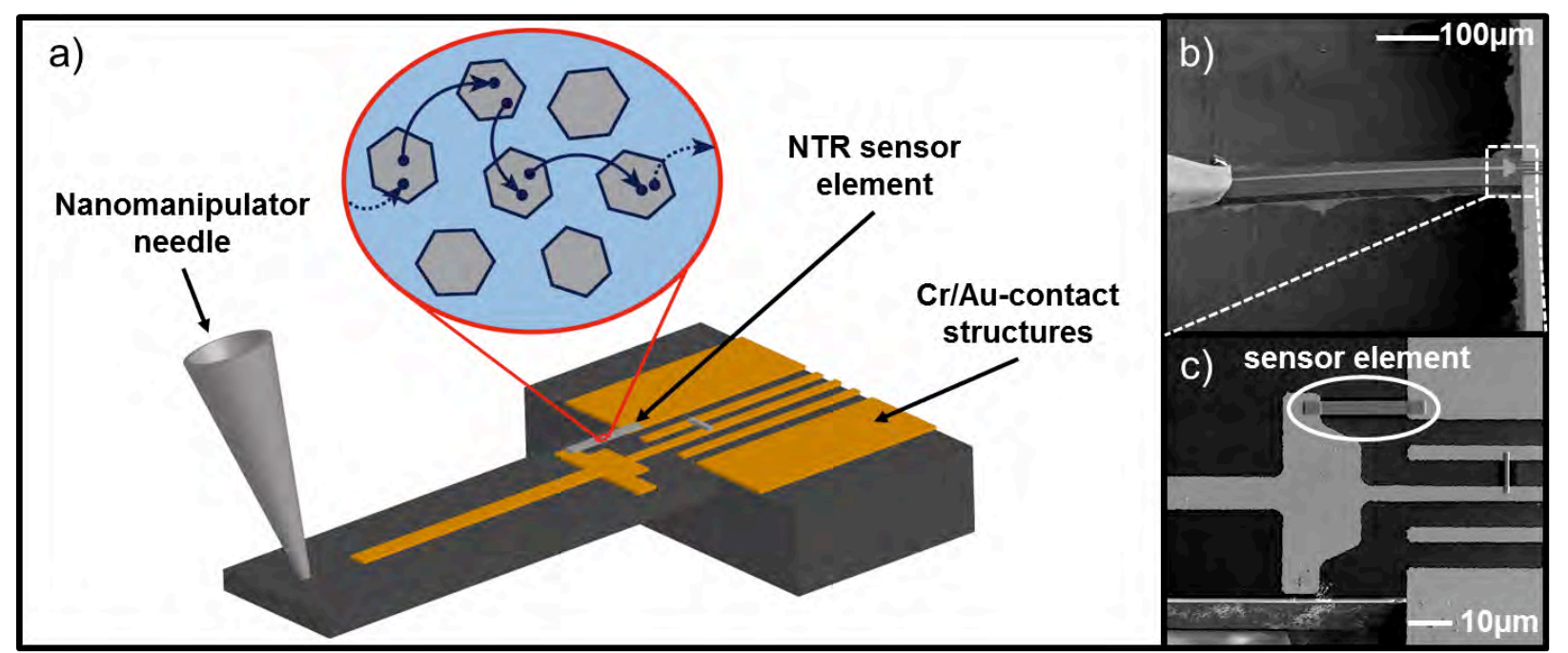

Figure 2: (a) Schematic of the experimental setup used for deflection measurements of the NTR sensor elements. The NTR sensor elements are deposited between Cr/Au-contacts at the bending edge of a cantilever structure. A deflection of the cantilever is induced by a nanomanipulator needle. Inset: Cartoon of the nanogranular structure of the NTR sensor elements (b) SEM image of the experimental setup showing the cantilever and nanomanipulator needle (left side). The dimensions of the cantilever are length: 500 $\mu \mathrm{m}$, width: $70 \mu \mathrm{m}$, height: $10 \mu \mathrm{m}$. (c) SEM image of the deposited NTR sensor element on the cantilever.

The precursor used for the FEBID process are typically metal-organic compounds that can contain a wide variety of metallic complexes (e.g. Au, Pt, W, Co, ...). For the passivation of the NTR sensor elements silicon-based precursor gases can be used. The sensitivity of the NTRs was analyzed using a cantilever substrate that was pre-patterned with $120 \mathrm{~nm}$ thick $\mathrm{Cr} / \mathrm{Au}$ contacts defined by UV photolithography. For the cantilever fabrication the starting material is a SOI wafer with $300 \mathrm{~nm}$ thick super low stress Si-Nitride layers on both sides of the polished wafer. A $600 \mathrm{~nm}$ thermal SiO layer separates the $10 \mu \mathrm{m}$ thick top Si layer from the bulk Si. After the pre-patterning of the $\mathrm{Cr} / \mathrm{Au}$-contacts a combination of reactive ion etching and anisotropic Si etching (using $\mathrm{KOH}$ solution) is used for the preparation of the cantilever structure. During the FEBID deposition process of the NTRs their conductance was measured in situ at fixed bias voltage using a source meter (Keithley 2400). The sensitivity of the NTR sensor elements was measured by inducing a deflection of the cantilever structure using a closed-loop nanomanipulator (SmarAct SLC1720-s) that is integrated inside the electron microscope chamber. Using a Wheatstone bridge setup the relative change in resistance, due to the deflection, was measured with a lock-in amplifier (Stanford Research SR830). Figure 2a displays the experimental setup and the cantilever structure used for the deflection measurements. In Figure 2c a SEM image of a NTR sensor element deposited between the $\mathrm{Cr} / \mathrm{Au}$-contacts at the bending edge of the cantilever is shown. For the fabrication of the ultrasmall cantilevers the starting material is a $\mathrm{Si}$ wafer with a $300 \mathrm{~nm}$ low-stress $\mathrm{Si}_{3} \mathrm{~N}_{4}$ layer on both sides. Using again a combination of reactive ion etching and anisotropic Si etching (using $\mathrm{KOH}$ solution) a $4 \times 4 \mathrm{~mm}^{2} \mathrm{Si}_{3} \mathrm{~N}_{4}$ membrane is produced with a thickness of $300 \mathrm{~nm}$. Afterwards FIB etching inside the microscope is used to cut out the cantilever structures from the $\mathrm{Si}_{3} \mathrm{~N}_{4}$ membrane. The same membrane structures were also used for pressure measurements in the low-vacuum regime. For this application the $\mathrm{Si}_{3} \mathrm{~N}_{4}$ membrane is covered with a thin Au-layer on 
the backside (thickness $\sim 20 \mathrm{~nm}$ ) that serves as a permeation barrier and improves the temperature stability. Additionally pressure measurements were performed on a $3.5 \times 3.5 \mathrm{~mm}^{2} \mathrm{Si}$ membrane structure with a thickness of $5 \mu \mathrm{m}$ that was pre-patterned with $\mathrm{Cr} / \mathrm{Au}$-contacts. The measurements were carried out in a high-vacuum chamber using a Pirani gauge as reference.

\section{Results and Discussion}

\section{NTR strain resistance effect}

In order to measure the strain resistance effect of the NTR sensor elements and calculate the corresponding gauge factor deflection measurements on a test cantilever structure were performed which is shown in Figure 2. The gauge factor represents the relative change in resistance normalized to the relative length change in the sensor element (gauge factor $k=(\Delta R / R) /(\Delta L / L)$. The NTR sensor structures are deposited between the Cr/Au-contacts at the bending edge of the cantilever (Figure 2c) with the dimensions: width $3 \mu \mathrm{m}$, length $15 \mu \mathrm{m}$. During the FEBID process we are able to measure the increase of the conductivity of the NTR sensor elements during deposition, which enables us to systematically study the influence of the conductivity on the sensitivity of our NTR sensor elements. The conductivity of the NTR sensor elements can be changed by: 1) gradually increasing the thickness of the Pt-C deposits and therefore increasing the volume of the conducting material, 2) Post-irradiation of the Pt-C deposits with the electron-beam of the SEM, 3) Passivation of the Pt-C deposits with a siliconbased precursor.
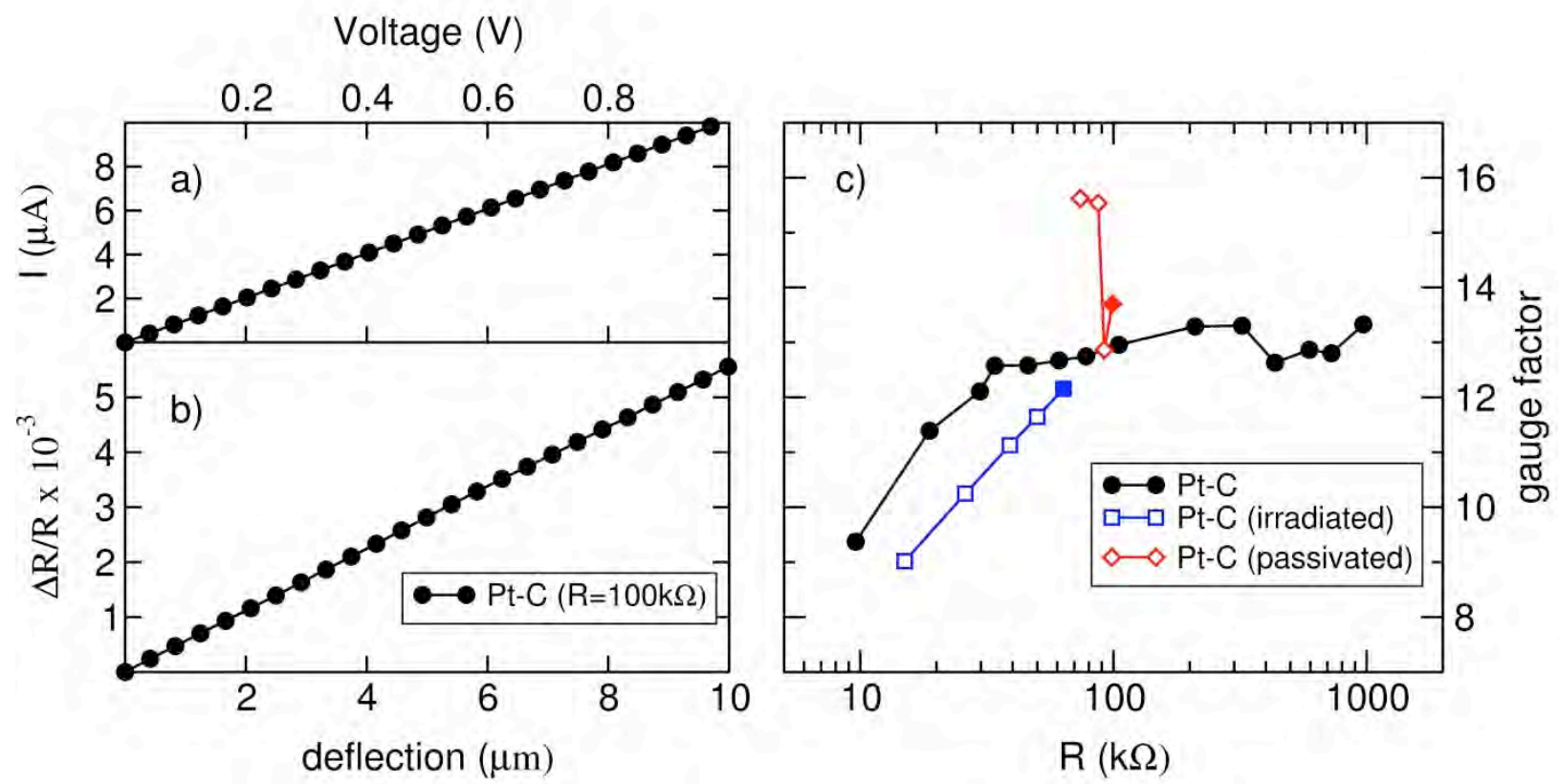

Figure 3: (a) Exemplary I(V)-characteristic for deposited Pt-C NTR sensor element. (b) Exemplary $\Delta R / R$ vs. cantilever deflection curve used for measuring the deflection sensitivity of a Pt-C NTR sensor element with a resistance of $100 \mathrm{k} \Omega$. Measurements were performed with a bias voltage of $100 \mathrm{mV}$. (c) Measured gauge factors as a function of resistance for Pt-C deposits during growth deposition (black circles), for postirradiated Pt-C deposits (blue squares) and for Pt-C deposits passivated with a silicon-based precursor (red diamonds).

After each deposition, post-irradiation, or passivation step we can measure the I(V)characteristic of the sensor elements. Figure 3a displays exemplarily the I(V)-characteristic for a $\mathrm{Pt}-\mathrm{C}$ sensor element with a resistance of $100 \mathrm{k} \Omega$. As can be seen the behavior is highly linear. Afterwards a nanomanipulator needle is used to induce a deflection of the cantilever and simultaneously measure the relative change in resistance of the NTR sensor elements. Figure 
3b shows a deflection measurement for a Pt-C deposit for a maximum deflection of $10 \mu \mathrm{m}$. Again the measured signal is highly linear and reproducible. Using the deflection measurements the gauge factors for NTR sensor elements can be calculated. Figure $3 \mathrm{c}$ shows the gauge factors for measurements where we increased the thickness of the Pt-C deposits, and therefore decreased the resistance (black circles). The gauge factor stays nearly constant at values of 1213 for resistances ranging from $1 \mathrm{M} \Omega$ down to $30 \mathrm{k} \Omega$. Below $30 \mathrm{k} \Omega$ the gauge factor shows a steeper decrease leading to a gauge factor of 9.4 at $10 \mathrm{k} \Omega$. In order to study the influence of electron-beam post-irradiation on the gauge factor of the NTR sensor elements, the growth of the deposits was terminated at $67 \mathrm{k} \Omega$. After the deposition the precursor flux was switched off and the system was pumped for more than two hours in order to guarantee that no residual precursor molecules were present in the chamber. Before starting the post-irradiation we measured the gauge factor of the sensor element. The starting gauge factor (Figure 3c, solid blue square) is in good agreement with thickness dependent gauge factors. For the irradiation of the deposits the same parameters for the electron-beam were used as for the previous deposition process only with switched off precursor flux. The irradiation was stopped at the targeted resistance and the gauge factor was measured. Figure $3 c$ shows the gauge factors for the post-irradiated deposits (open blue squares). The irradiation induces a decrease in resistance accompanied with a decrease in the gauge factor that leads to lower gauge factors compared to those obtained for $\mathrm{Pt}-\mathrm{C}$ deposits with increasing thickness. The change in resistance and gauge factor for sensor elements with increased thickness as well as after postirradiation is mainly triggered by two mechanisms. First, electron irradiation of the as-grown deposit induces a reduction of the thickness due to carbon removal from the $\mathrm{Pt}-\mathrm{C}$ sensor elements.[14] The origin of carbon removal can be attributed to local heating[15, 16] and knockon effects.[17] This leads to a relative increase of the conductivity. Second, the irradiation induces a structural change in the Pt-C deposits. Using micro-Raman spectroscopy and transmission electron microscopy (TEM) one can observe graphitization of the carbonaceous matrix accompanied with an increase of crystallinity of the platinum nano-crystals.[14] Electron transport measurements reveal a variation of the tunnel coupling, explaining the alteration of the gauge factors.[14, 18] The change in resistance and gauge factor is more pronounced for the post-irradiated sensor elements, due to the fact that no new material is deposited during the irradiation process in contrast to the measurements with increasing thickness.

Additionally we studied the influence of a passivation layer using a silicon-based precursor. Again the deposition of the Pt-C was terminated at $100 \mathrm{k} \Omega$ and the gauge factor prior to the passivation was measured (Figure $3 \mathrm{c}$ solid red diamond). Subsequently the $\mathrm{Pt}-\mathrm{C}$ deposits were passivated with the silicon-based precursor. The passivation process was stopped after different times and the gauge factors were determined. As can be seen in Figure 3c (open red diamonds) the passivation of the $\mathrm{Pt}-\mathrm{C}$ deposits also leads to a decrease in resistance but on a much longer timescale compared to the post-irradiation (not shown here). Interestingly the gauge factor shows a small decrease in the beginning of the passivation process, however for longer passivation times we observe an increase of $\sim 25 \%$ to gauge factors of 16 . The increase in conductivity and sensitivity for the passivated Pt-C deposits can be attributed to an alteration of the dielectric matrix. Presumably silicon is embedded into the carbonaceous matrix, therefore changing the dielectric constant of the matrix and the coupling strength for the nanogranular Ptcrystals. In addition to the increase in sensitivity the amorphous silicon passivation also leads to an improved long-term stability under ambient conditions. Whereas the bare Pt-C deposits show a small almost linear aging in air, the passivated deposits are extremely stable even after several months.

The recent reachable gauge factors of 16 have great potential for further optimization. Based on recent advances in the theory of electronic transport in nanogranular metals a systematic approach is available to increase the gauge factors significantly.[12] For a reduction of the grain size of the metallic nanoparticles as well as insulating matrices with small dielectric constants gauge factors larger than 50 are predicted. Supported are these theoretical predictions by recent experimental results using nickel-containing DLC-layers, where gauge factors $\geq 30$ could be directly obtained.[19] 
Applications: Ultra-small cantilevers

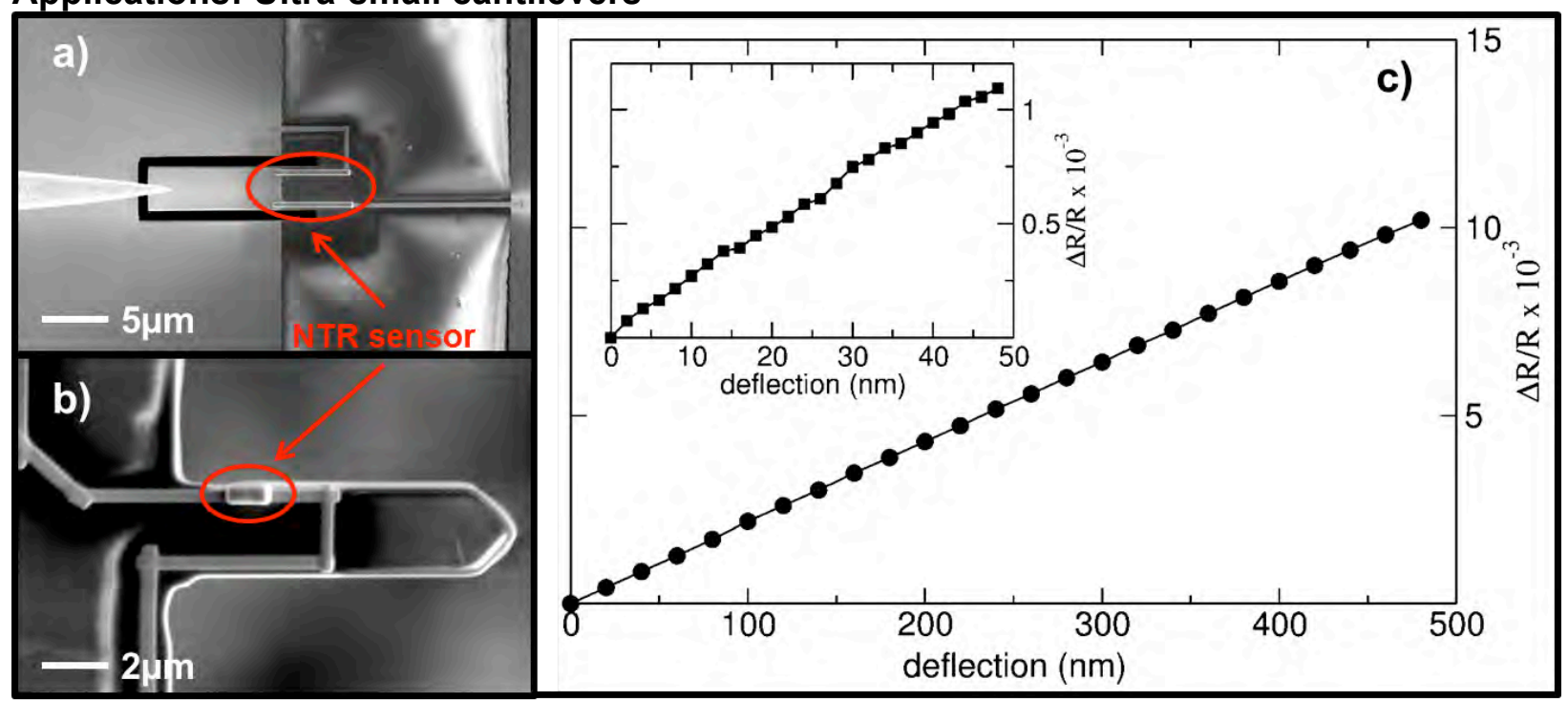

Figure 4: (a) + (b) SEM image of micro-cantilever structures with dimensions: a) $20 \mu \mathrm{m} \times 4 \mu \mathrm{m} \times 0.3 \mu \mathrm{m}$ and b) $10 \mu \mathrm{m} \times 2 \mu \mathrm{m} \times 0.5 \mu \mathrm{m}$. The cantilever structures were fabricated by FIB etching of thin $\mathrm{Si}_{3} \mathrm{~N}_{4}$-membranes and afterwards outfitted with NTR sensor elements. In (a) also the nanomanipulator needle is shown that is used for deflection measurements. (c) $\Delta R / R$ vs. cantilever deflection curve used for measuring the deflection sensitivity of a Pt-C NTR sensor element for the micro-cantilever structure shown in (a). Measurements were performed with a bias voltage of $1 \mathrm{~V}$. Inset: deflection measurement for a maximum deflection of $\Delta \mathrm{z}=50 \mathrm{~nm}$.

One promising application for the NTR sensor elements are ultra-small cantilevers for atomic force microscopy (AFM) applications. A main drawback of AFM is the low throughput. Due to the scanning nature of the image acquisition it takes several minutes for a high resolution AFM image, therefore a lot of effort has been dedicated in recent years to increase the speed of AFM. One necessity for increasing the speed of AFM is the decrease of cantilever size and thickness. Conventional standard cantilevers have dimensions of 30-100 $\mu \mathrm{m}$ width, $60-200 \mu \mathrm{m}$ length, and 0.5-5 $\mu \mathrm{m}$ thickness. Recent developments allow for a reduction of cantilever dimensions making small cantilevers commercially available with dimensions: width $<10 \mu \mathrm{m}$, length $10-30 \mu \mathrm{m}$, thickness $0.1-0.3 \mu \mathrm{m}$. Nevertheless, in case of the small AFM cantilevers, the dimensions of the cantilever is comparable to the minimum spot size that the laser beam (used for measuring of the cantilever deflection) can be focused down to, due to the diffraction limit. Therefore, further decreasing the size of the cantilever is not easily possible using optical detection. Also conventional piezoresistive sensor elements offer no way to circumvent this problem, since the piezoresistive sensor elements require a process-related minimal structural size of $\sim 30 \mu \mathrm{m}$ in length and $\sim 2 \mu \mathrm{m}$ in thickness. A further increase in the performance of the cantilevers (both in sensitivity and speed) is only possible if a different cantilever deflection detection scheme is developed for even smaller cantilevers. Due to the high resolution of the FEBID process, NTR sensor structures with dimensions well below $50 \mathrm{~nm}$ are easily achievable. Therefore, NTR sensor elements offer a new possibility for the development and fabrication of ultra-small cantilevers. Figure 4 displays a proof of principle study for NTR cantilevers. In Figure 4a + b SEM images of micro-cantilever structures are shown, which were fabricated by FIB etching of thin $\mathrm{Si}_{3} \mathrm{~N}_{4}$-membranes. After the etching step NTR sensor elements and contact structures were deposited on the cantilever. As can be seen in Figure $4 \mathrm{~b}$ the cantilever has the dimensions of $10 \mu \mathrm{m}$ length, $2 \mu \mathrm{m}$ width, $0.5 \mu \mathrm{m}$ thickness, whereas the NTR sensor element itself has the dimensions of $1 \mu \mathrm{m}$ length and $0.2 \mu \mathrm{m}$ width. Measurements of $\Delta R / R$ vs. cantilever deflection were performed on these cantilever structures, revealing a highly linear and reproducible signal (Figure 4c) for a maximum deflection of $\Delta z=500 \mathrm{~nm}$. Even for a deflection of only $50 \mathrm{~nm}$ (Figure 4c inset) the deflection measurement shows a linear response with an overall resolution of $\sim 1 \mathrm{~nm}$. But these results are by far not the ultimate limit of the NTR cantilevers concerning dimensions of the cantilever as well as deflection sensitivity (see chapter "Noise \& Sensitivity estimations" for further details). 


\section{Noise \& Sensitivity estimations}

An important parameter for cantilever-based (as well as membrane-based) sensors is the minimum detectable deflection. For a cantilever with conventional piezoresistive or NTR-based readout, the minimum detectable deflection depends not only on the deflection sensitivity of the piezoresistor or the NTR, but is mainly limited by the noise level. The deflection sensitivity for a rectangular cantilever beam, which relates the relative change in resistance $\Delta R / R$ to the cantilever deflection $\Delta z$, can be expressed as

$$
\left(\frac{\Delta R}{R}\right) \frac{1}{\Delta z}=K \frac{3\left(l-\frac{1}{2} L\right) t}{2 l^{3}} .
$$

Here $K$ is the gauge factor of the sensor element, $I$ is the cantilever length, $t$ is the cantilever thickness and $L$ the length of the sensor element.[20] It is a common held assumption that a large gauge factor will serve to maximize a displacement sensor's performance, but actually this is only one element in optimizing its output. Cantilever dimensions and noise level of the sensor elements however become the main parameters for sensor optimization for materials scaled down to nanometer dimensions. For the micro-cantilever displayed in Figure $4 \mathrm{~b}$ we can calculate the resistance change for a cantilever deflection of $\Delta z=1 \mathrm{~nm}$, a gauge factor of $K=15$, and a resistance for the NTR sensor element of $R_{N T R}=10 \mathrm{k} \Omega$, using equation (1). This results in a resistance change of $\Delta R=1.1 \Omega$. Using a Wheatstone bridge setup the output voltage $V_{G}$ is given by

$$
V_{G}=\left(\frac{R+\Delta R}{2 R+\Delta R}-\frac{1}{2}\right) \cdot V_{\text {bias }}
$$

where $V_{\text {bias }}=1 \mathrm{~V}$ is the used bias voltage for the deflection measurements. Therefore, we get an output voltage of $V_{G}=26.7 \mu \mathrm{V}$ and a deflection sensitivity of $26.7 \mu \mathrm{V} / \mathrm{nm}$. The resonance frequency $f_{r}$ for the $\mathrm{Si}_{3} \mathrm{~N}_{4}$ cantilever can be calculated using

$$
f_{r}=\frac{1.02}{2 \pi} \frac{t}{l^{2}} \sqrt{\frac{E}{\rho}} .
$$

$E$ and $\rho$ represent the E-module and the mass density. For our cantilever (Figure $4 \mathrm{~b}$ ) we get a resonance frequency of $f_{r}=7 \mathrm{MHz}$. In recent experiments it could be shown that the noise in Pt$C$ based NTR sensor elements follows a $1 / f$ behavior with a crossing of the Johnson noise level at a frequency of $f_{c} \sim 1 \mathrm{kHz}$.[13] If we now consider a desired bandwidth of our cantilever around the resonance frequency $f_{r}$, the noise of the NTR sensor element is solely dominated by the thermal noise level. The effective noise density can be expressed as

$$
U_{\theta f f}^{R}=\sqrt{4 k_{B} T R \Delta f} .
$$

Considering a desired bandwidth for our cantilever of at least $200 \mathrm{kHz}$ we can calculate an estimated noise using eq. (4) of $U_{\theta f f}^{R}=5.8 \mu \mathrm{V}$. This corresponds to a noise level of $\sim 0.2 \mathrm{~nm}$. For realistic amplitudes for AFM imaging of 1 to $10 \mathrm{~nm}$, this leads to a Signal-to-Noise ratio (SNR) of 5 to 50 for the cantilever structure shown in Figure 4b. This SNR can be much improved taking into the account the advantages of the FEBID technique. Due to the high resolution of the FEBID process, much smaller and thinner cantilever structures can be equipped with the NTR sensor elements. If one considers a $\mathrm{Si}_{3} \mathrm{~N}_{4}$ cantilever with the dimensions: length $1 \mu \mathrm{m}$, width $0.5 \mu \mathrm{m}$, thickness $0.05 \mu \mathrm{m}$ and a resistance of the NTR sensor element of $R=1 \mathrm{k} \Omega$, the deflection sensitivity would be $210.9 \mu \mathrm{V} / \mathrm{nm}$ with a resonance frequency of $f_{r}=70 \mathrm{MHz}$. This would lead to an effective noise level of $U_{\text {off }}^{R}=4.1 \mu \mathrm{V}$ or $0.02 \mathrm{~nm}$ at a bandwidth of $1 \mathrm{MHz}$. Such a cantilever would clearly outperform all existing optical cantilevers in terms of resolution and achievable scanning speeds. 
Applications: Pressure membranes

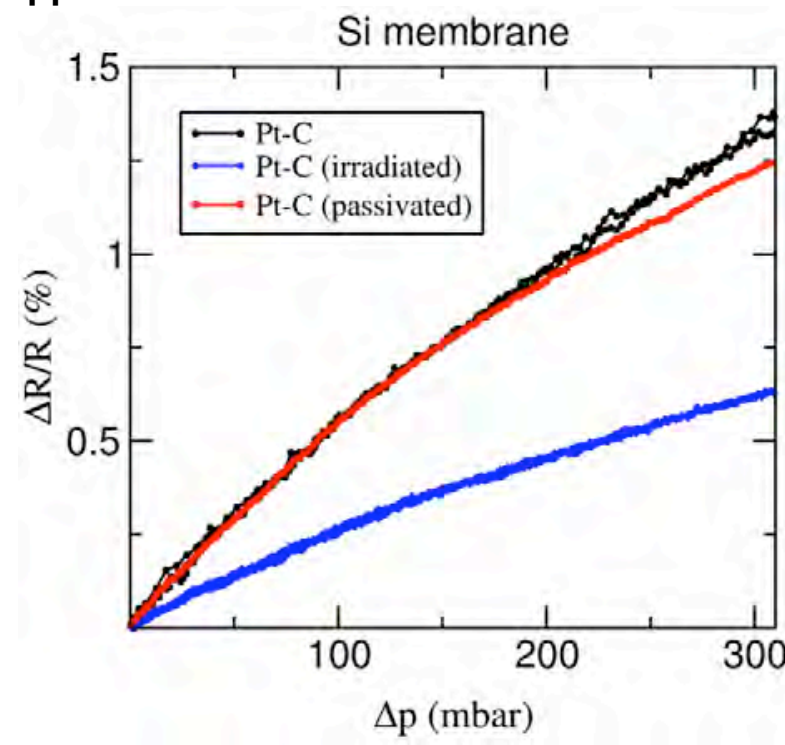

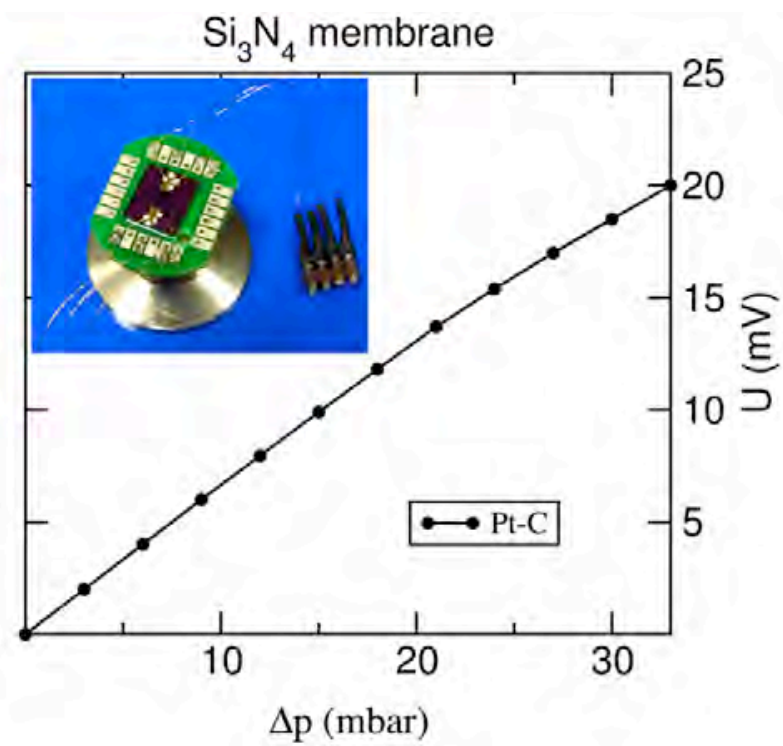

Figure 5: (Left) Pressure measurement for Si membrane with the dimensions $3.5 \times 3.5 \mathrm{~mm}^{2}$ and a thickness of $\sim 5 \mu \mathrm{m}$. Measurements were performed with different NTR sensor elements: bare Pt-C (black circles, $\mathbf{R}=$ $730 \mathrm{k} \Omega$ ), irradiated Pt-C (blue diamonds, $R=8 \mathrm{k} \Omega$ ), and passivated Pt-C (red squares, $R=14 \mathrm{k} \Omega$ ). All measurements were done bidirectional and show only little hysteresis during the measurement. (Right) Pressure measurement for $\mathrm{Si}_{3} \mathrm{~N}_{4}$ membrane with the dimensions $4 \times 4 \mathrm{~mm}^{2}$ and a thickness of $300 \mathrm{~nm}$. Measurements were performed with a bare Pt-C deposit with $\mathrm{R}=100 \mathrm{k} \Omega$. The inset shows the pre-production model that was used for the measurements.

In a second application we used the NTR sensor elements for pressure measurements on Siand $\mathrm{Si}_{3} \mathrm{~N}_{4}$-membranes. Figure 5 left displays the measurements for a $\mathrm{Si}$ membrane with the dimensions $3.5 \times 3.5 \mathrm{~mm}^{2}$ and thickness of $\sim 5 \mu \mathrm{m}$ for a maximum pressure difference of $\Delta p=300$ mbar. Different NTR sensor elements were used for the measurements. The bare Pt-C sensor elements (Figure 5 left, black circles) show a high sensitivity with an observable noise that can be attributed to the relatively high resistance of the sensor element $(R=730 \mathrm{k} \Omega)$. On the contrary the post-irradiated NTR sensor element (Figure 5 left, blue diamonds) shows both smaller sensitivity and noise. The best performance can be achieved using the NTR sensor elements that are passivated with a silicon-based precursor (Figure $5 \mathrm{left}$, red squares). The sensitivity is equally high as compared to the bare $\mathrm{Pt}-\mathrm{C}$ deposits, but due to the lower resistance $(R=14 \mathrm{k} \Omega)$ the overall noise is much smaller. Also the long-term stability of the passivated NTR sensor elements is an additional advantage. These results are in good agreement with the results for the gauge factor measurements shown in Figure $3 \mathrm{c}$.

A specific strength of the NTR sensor elements in combination with the FEBID technique, especially compared to conventional piezoresistive sensor elements, is the adaptability to very thin membrane structures as well as the independence of substrate material. As a demonstration we used a $4 \times 4 \mathrm{~mm}^{2}$ and $300 \mathrm{~nm}$ thick $\mathrm{Si}_{3} \mathrm{~N}_{4}$-membrane (see inset Figure 5) and deposited NTR sensor elements at the bending edge of the membrane. Also for this membrane structure, which cannot be operated with conventional piezoresistors, we are able to measure a highly reproducible signal using the bare Pt-C NTR sensor elements. In contrast to conventional Pirani gauges our NTR pressure sensor is gas-type independent allowing a calibration free measurement even under coarse vacuum conditions. In addition, the NTR pressure sensor should exhibit an extended life-time compared to cold-cathode ionization gauges since no cathode abrasion will occur.

Due to the new degrees of freedom, concerning membrane thickness and material, which are usable with the NTR sensor elements, optimized membrane structures are feasible for a variety of applications. Especially applications in the low- and high-vacuum regime using thin $\mathrm{Si}_{3} \mathrm{~N}_{4^{-}}$ membranes, applications in the medium and high pressure regime using steel membranes, as well as miniaturized pressure sensors for special applications, e.g., catheter pressure sensors can be realized. 


\section{Conclusion}

In summary, a novel type of three-dimensional strain sensing element based on nanogranular metals has been demonstrated using the FEBID technique. In contrast to the established process for the fabrication of conventional piezoresistive sensors, which require a complex fabrication procedure, our process is a mask-less single-step procedure making it readily usable for rapid prototyping. It is not limited to silicon-based substrates and can be easily applied to nearly all solid surfaces, even with highly sophisticated geometrical shapes. In addition, the high resolution of the FEBID technique allows an easy down-scaling of sensor structures well below $20 \mathrm{~nm}$, which opens new pathways towards highly miniaturized sensor elements in combination with a relatively simple fabrication process that can be used for real 3D-structuring on a wide range of different substrate materials and environments. We can tune the resistance and the gauge factors of the NTR sensor elements by increasing the thickness of the deposits, by postirradiation or by passivation, resulting in highly reproducible gauge factors of $K=13$ for bare Pt$\mathrm{C}$ and $K=16$ for passivated Pt-C. The actual achievable gauge factors have a great potential for further optimization. Additionally, we showed the applicability of the NTR sensor elements for ultra-small cantilevers and very thin pressure membranes. Here, the ultra-small cantilevers show the potential to clearly outperform the existing optical cantilevers in terms of resolution and achievable scanning speeds. For the pressure membranes the new degrees of freedom, concerning membrane thickness and material, allow the realization of novel pressure sensors which are not feasible so far.

In conclusion, the methodology presented here promises to be suitable for different applications of force based sensors, ranging from simple strain and stress sensing to advanced applications in MEMS- and NEMS devices (e.g. automotive and aviation, measurement and control technology), life sciences and biomedical applications (e.g. DNA-analysis, gas- and fluid analysis, single protein analysis, drug screening, artificial nose and more) with the potential for exciting new sensor products. A specific strength of the NTR-technology is that it is easily adaptable to the most varied problems. The independence of the used substrate, the mask-less single-step process in combination with the high resolution of the sensor structures enables a rapid and cost-efficient adaptability to a vast area of appliances.

\section{Acknowledgements}

The authors acknowledge financial support by the NanoNetzwerkHessen (NNH) and the European FP7 project "ALBICAN" under Grant No. 286146. Financial support by the BeilsteinInstitut, Frankfurt/Main, Germany within the research collaboration NanoBiC is also gratefully acknowledged.

\section{References}

[1] M. Zougagh and A. Rios. Micro-electromechanical sensors in the analytical field. Analyst, 134(7):1274-1290, 2009.

[2] M. Alvarez and L. M. Lechuga. Microcantilever-based platforms as biosensing tools. Analyst, 135(5):827-836, 2010.

[3] T. Takeno, T. Takagi, A. Bozhko, M. Shupegin, and T. Sato. Metal-containing diamond-like nanocomposite thin film for advanced temperature sensors, volume 475-479. Trans Tech Publications Ltd, 2005.

[4] R. Koppert, D. Goettel, O. Freitag-Weber, and G. Schultes. Nickel containing diamond like carbon thin films. Solid State Sci., 11(10):1797-1800, October 2009.

[5] J. Cao, Q. Wang, and H. J. Dai. Electromechanical properties of metallic, quasimetallic, and semiconducting carbon nanotubes under stretching. Physical Review Letters, 90(15):157601, April 2003.

[6] C. Hierold, A. Jungen, C. Stampfer, and T. Helbling. Nano electromechanical sensors based on carbon nanotubes. Sensors and Actuators A-physical, 136(1):51-61, May 2007. 
[7] M. Menon, D. Srivastava, I. Ponomareva, and L. A. Chernozatonskii. Nanomechanics of silicon nanowires. Physical Review B, 70(12):125313, September 2004.

[8] J. Zhou, Y. D. Gu, P. Fei, W. J. Mai, Y. F. Gao, R. S. Yang, G. Bao, and Z. L. Wang. Flexible piezotronic strain sensor rid d-5536-2009 rid e-2176-2011. Nano Letters, 8(9):3035-3040, September 2008.

[9] C. H. Jin, J. Y. Wang, Q. Chen, and L. M. Peng. In situ fabrication and graphitization of amorphous carbon nanowires and their electrical properties rid e-2089-2011. Journal of Physical Chemistry B, 110(11):5423-5428, March 2006.

[10] J. D. Wnuk, J. M. Gorham, and D. H. Fairbrother. Growth and microstructure of nanoscale amorphous carbon nitride films deposited by electron beam irradiation of 1,2-diaminopropane. Journal of Physical Chemistry C, 113(28):12345-12354, July 2009.

[11] H. Gerdes, R. Bandorf, U. Heckmann, V. Schmidt, H. U. Kricheldorf, and G. Brauer. Sputter deposition of strain gauges using ito/ag. Plasma Processes Polym., 6:S813-S816, 2009.

[12] M. Huth. Granular metals: From electronic correlations to strain-sensing applications. J. Appl. Phys., 107(11):113709, June 2010.

[13] C. H. Schwalb, C. Grimm, M. Baranowski, R. Sachser, F. Porrati, H. Reith, P. Das, J. Muller, F. Volklein, A. Kaya, and M. Huth. A tunable strain sensor using nanogranular metals. Sensors, 10(11):9847-9856, November 2010.

[14] F. Porrati, R. Sachser, C. H. Schwalb, A. S. Frangakis, and M. Huth. Tuning the electrical conductivity of pt-containing granular metals by postgrowth electron irradiation. Journal of Applied Physics, 109(6):063715, March 2011.

[15] A. Botman, J. J. L. Mulders, R. Weemaes, and S. Mentink. Purification of platinum and gold structures after electron-beam-induced deposition. Nanotechnology, 17(15):3779-3785, August 2006.

[16] S. J. Randolph, J. D. Fowlkes, and P. D. Rack. Effects of heat generation during electron-beaminduced deposition of nanostructures. Journal of Applied Physics, 97(12):124312, June 2005.

[17] F. Banhart. Irradiation effects in carbon nanostructures. Reports On Progress In Physics, 62(8):1181-1221, August 1999.

[18] R. Sachser, F. Porrati, C. H. Schwalb, and M. Huth. Universal conductance correction in a tunable strongly coupled nanogranular metal. Physical Review Letters 107, 206803 November 2011.

[19] R. Koppert, D. Gottel, G. Schultes, and U. Werner. Nanoni@c: Supersensitive functional layer for pressure and force sensors. Tm-technisches Messen, 77(12):631-637, 2010.

[20] J. A. Harley and T. W. Kenny. 1/f noise considerations for the design and process optimization of piezoresistive cantilevers. 9(2):226-235, June 2000. 